\title{
Descrição morfológica do plexo braquial de jaguatirica (Leopardus pardalis)
}

\author{
Kylma Lorena Saldanha Chagas ${ }^{1}$ \\ Lara Cochete Moura Fé ${ }^{1}$ \\ Luiza Correa Pereira ${ }^{2}$ \\ Érika Branco ${ }^{1 *}$ \\ Ana Rita de Lima ${ }^{1}$ \\ ${ }^{1}$ Laboratório de Pesquisa Morfológica Animal, Universidade Federal Rural da Amazônia \\ Avenida Presidente Tancredo Neves, 2501, CEP 66077-530, Belém - PA, Brasil \\ ${ }^{2}$ Área de Mina Bauxita, Vale Paragominas - PA, Brasil \\ * Autor para correspondência \\ ebranco.ufra@gmail.com
}

Submetido em 16/08/2013

Aceito para publicação em 12/03/2014

\section{Resumo}

O plexo braquial é constituído por raízes ventrais dos nervos espinhais que se unem para a formação dos troncos nervosos. É geralmente formado por contribuições dos três últimos nervos cervicais e dos dois primeiros nervos torácicos. Devido à escassez de informações relativas à neuroanatomia, objetivou-se buscar a descrição da morfologia macroscópica do plexo braquial da jaguatirica (Leopardus pardalis). Foram utilizados dois exemplares de jaguatirica provenientes da área de Mina Bauxita Paragominas - PA, Brasil/Empresa Terra LTDA, doados após morte por atropelamento ao Laboratório de Pesquisa Morfológica Animal (LaPMA) da Universidade Federal Rural da Amazônia (UFRA), os quais foram fixados em solução aquosa de formoldeído a $10 \%$ por via intramuscular. Após fixação os animais foram dissecados, possibilitando a observação e identificação dos ramos ventrais dos nervos espinhais cervicais e torácicos que participaram da formação do plexo braquial. O plexo braquial mostrou-se formado por quatro troncos, os quais originaram os ramos ventrais dos nervos espinhais cervicais $\mathrm{C}_{6}, \mathrm{C}_{7}$ e $\mathrm{C}_{8}$ e do primeiro torácico $\mathrm{T}_{1}$. Tais troncos deram origem aos nervos supraescapular, subescapular, musculocutâneo, axilar, radial, mediano, ulnar, toracodorsal e torácico lateral.

Palavras-chave: Jaguatirica; Nervos; Plexo braquial

\section{Abstract}

Morphological description of the brachial plexus in ocelot (Leopardus pardalis). The brachial plexus is formed by the ventral roots of the spinal nerves, which unite to form the nerve trunks. It is usually formed by contributions of the last three cervical nerves and the first two thoracic nerves. Due to the scarcity of information on neuroanatomy, this study aimed to determine the macroscopic morphology of the brachial plexus of the ocelot (Leopardus pardalis). In this work, we used two ocelot specimens from the area of the Paragominas Bauxite Mine, PA, Brazil/Empresa Terra LTDA, with permission from SEMA - BP Nos. 455/2009 and 522/2009. The animals were donated to the Research Laboratory of Animal Morphology (LaPMA), Federal Rural University 
of Amazonia (UFRA), after they were accidentally run over. They were fixed by intramuscular injection of $10 \%$ formaldehyde. After fixation, the animals were dissected, allowing visualization of the thoracic nerves, as well as the identification of the ventral rami of the cervical and thoracic spinal nerves forming the brachial plexus. The brachial plexus was found to be formed by four trunks, which originated the ventral branches of cervical spinal nerves $\mathrm{C} 6, \mathrm{C} 7$ and $\mathrm{C} 8$ and the first thoracic (T1). These trunks gave rise to the suprascapular, subscapular, musculocutaneous, axillary, radial, median, ulnar, thoracodorsal and lateral thoracic nerves.

Key words: Brachial plexus; Nerves; Ocelot

\section{Introdução}

A jaguatirica é um felino carnívoro de habitat variado: Cerrado, Caatinga, Pantanal, e, principalmente florestas tropicais e subtropicais, sendo encontrada também no continente americano desde o sudoeste do Texas e oeste do México até o norte da Argentina. No Brasil, ocorre em todas as regiões, à exceção do Rio Grande do Sul (OLIVEIRA; CASSARO, 1999).

Considerado o maior dos pequenos felinos pintados, seu comprimento varia de 50 a $80 \mathrm{~cm}$, mais 20 a $40 \mathrm{~cm}$ da cauda, podendo pesar de 4 a $11 \mathrm{~kg}$. Sua cor de pelagem é bastante variável, mesmo dentro de uma mesma população (MURRAY; GARDNER, 1997).

Nos vertebrados, o plexo braquial é constituído por raízes ventrais dos nervos espinhais que se unem para a formação dos troncos nervosos. A divisão cranial e a divisão caudal a esses troncos se reúnem e formam cordões nervosos, dos quais, constituídos os nervos periféricos, se dirigirem para as extremidades do membro torácico (MILLER, 1934; MILLER et al., 1964; DYCE et al., 2010; KÖNIG; LIEBICH, 2011) .

O plexo braquial supre quase todas as estruturas do membro torácico, exceto os músculos trapézio, omotransverso, braquiocefálico e romboide, bem como a pele sobre a região dorsal ao ombro. Geralmente é formado por contribuições dos três últimos nervos cervicais e dos dois primeiros torácicos, algumas vezes apresentando função também o quinto nervo cervical (BUDRAS et al., 2012).

O plexo passa entre as partes do músculo escaleno e se divide em ramos periféricos, passando pela axila e destinando-se a diferentes locais. Tais ramos são compreendidos pelo nervo torácico longo que inerva o músculo serrátil ventral; nervo toracodorsal, que inerva o músculo grande dorsal; nervos peitorais cranial e caudal, para os músculos peitorais, incluindo o músculo subclávio; nervo subescapular inervando o músculo subescapular e o nervo torácico lateral para o músculo cutâneo do tronco e para a pele sobre a parte ventral do tórax e abdome (DYCE et al., 2010; BUDRAS et al., 2012).

Devido à escassez de informações relativas à neuroanatomia do Leopardus pardalis, e, tendo em vista a importância da anatomia sistêmica para a compreensão fisiológica da inervação do membro torácico e processo locomotor destes animais e das possibilidades de estudos de anatomia comparada, este trabalho tem como ambição a descrição sistêmica da morfologia macroscópica do plexo braquial da jaguatirica, bem como uma contribuição à neuroanatomia comparada na Medicina Veterinária.

\section{Material e Métodos}

Foram utilizados dois exemplares (um macho e uma fêmea) de Leopardus pardalis, adultos, provenientes da área de mina Bauxita Paragominas - PA, sob autorização SEMA - PA no 455/2009 e 522/2009, doados ao Laboratório de Pesquisa Morfológica Animal (LaPMA) da Universidade Federal Rural da Amazônia (UFRA) após morte por atropelamento. Estes espécimes ora criopreservados foram descongelados em água corrente por aproximadamente 4 horas e em seguida fixados com solução aquosa de formoldeído a $10 \%$ mediante infusão intramuscular, bem como em todas as cavidades, com posterior submersão desses animais nesta mesma solução, por um período mínimo de sete dias.

Após o período de fixação, os exemplares foram dissecados, realizando-se incisões próximas ao esterno, onde a pele e a musculatura foram rebatidas, até atingir o plexo braquial no espaço axilar. A desarticulação das costelas com retirada do esterno e completa evisceração da 
cavidade torácica possibilitou a observação e identificação dos ramos ventrais dos nervos espinhais cervicais e torácicos que participaram da formação do plexo braquial.

Toda nomenclatura adotada foi baseada na Nomenclatura Atômica Veterinária (INTERNATIONAL COMMITTEE ON VETERINARY GROSS ANATOMICAL NOMENCLATURE, 2012).

\section{Resultados}

Nos dois espécimes analisados a origem do plexo braquial foi advinda dos ramos ventrais dos nervos espinhais cervicais $\mathrm{C}_{6}, \mathrm{C}_{7}$ e $\mathrm{C}_{8}$ e do nervo torácico $\mathrm{T}_{1}$.
Nos animais analisados, os nervos encontrados participando da formação do plexo braquial foram: subclávio, supraescapular, subescapular, musculocutâneo, peitorais craniais, axilar, radial, mediano, ulnar, toracodorsal e torácico lateral.

O ramo cervical $\mathrm{C}_{6}$ deu origem aos nervos subclávio, supraescapular, subescapular, peitorais craniais e musculocutâneo. $\mathrm{O}$ ramo cervical $\mathrm{C}_{7}$ originou os nervos subclávio, supraescapular, subescapular, peitorais craniais, musculocutâneo, axilar, radial e mediano. $\mathrm{O}$ ramo cervical $\mathrm{C}_{8}$ deu origem aos nervos radial, mediano, ulnar, toracodorsal e torácico lateral, e por fim, o ramo $T_{1}$ originou os nervos radial, mediano e ulnar (Tabela 1; Figura 1).

TABELA 1: Origem dos nervos que compõe o plexo braquial nos espécimes analisados de Leopardus pardalis.

\begin{tabular}{|c|c|}
\hline Nervo & Origem \\
\hline Subclávio & Ramo ventral cervical $\mathrm{C}_{6}$ e $\mathrm{C}_{7}$ \\
\hline Supraescapular & Ramo ventral cervical $\mathrm{C}_{6}$ e $\mathrm{C}_{7}$ \\
\hline Subescapular & Ramos ventrais cervicais $\mathrm{C}_{6}$ e $\mathrm{C}_{7}$ \\
\hline Musculocutâneo & Ramos ventrais cervicais $\mathrm{C}_{6} \mathrm{e} \mathrm{C}_{7}$ \\
\hline Peitorais craniais & Ramo ventral cervical $\mathrm{C}_{6} \mathrm{e} \mathrm{C}_{7}$ \\
\hline Axilar & Ramos ventrais cervicais $\mathrm{C}_{7}$ \\
\hline Radial & Ramos ventrais cervicais $\mathrm{C}_{7}, \mathrm{C}_{8}$ e torácico $\mathrm{T}_{1}$ \\
\hline Mediano & Ramos ventrais cervicais $\mathrm{C}_{7}, \mathrm{C}_{8}^{\circ}$ e torácico $\mathrm{T}_{1}$ \\
\hline Ulnar & Ramo ventral cervical $\mathrm{C}_{8}$ e torácico $\mathrm{T}_{1}$ \\
\hline Toracodorsal & Ramo ventral cervical $\mathrm{C}_{8}$ \\
\hline Torácico Lateral & Ramo ventral torácico $\mathrm{C}_{8}$ \\
\hline
\end{tabular}


FIGURA 1: (A) Esquema representativo do plexo braquial da jaguatirica, que apresenta origem nos ramos ventrais cervicais $\mathrm{C}_{6}, \mathrm{C}_{7}$, e $\mathrm{C}_{8}$ e no ramo ventral torácico $T_{1}$, originando os nervos subclávio (1), supraescapular (2), subescapular (3), musculocutâneo (4), peitorais craniais (5), axilar (6), radial (7), mediano (8), ulnar (9), toracodorsal (10) e torácico lateral (11). (B) Fotomacrografia da face ventral do membro torácico esquerdo do espécime fêmea de Leopardus pardalis. Observar os ramos ventrais cervicais $\mathrm{C}_{6}, \mathrm{C}_{7}, \mathrm{e}_{8}$ e o ramo ventral torácico $\mathrm{T}_{1}$, originando os nervos supraescapular (1), subescapular (2), musculocutâneo (3), axilar (4), radial (5), mediano (6), ulnar (7), toracodorsal (8) e torácico lateral (9).
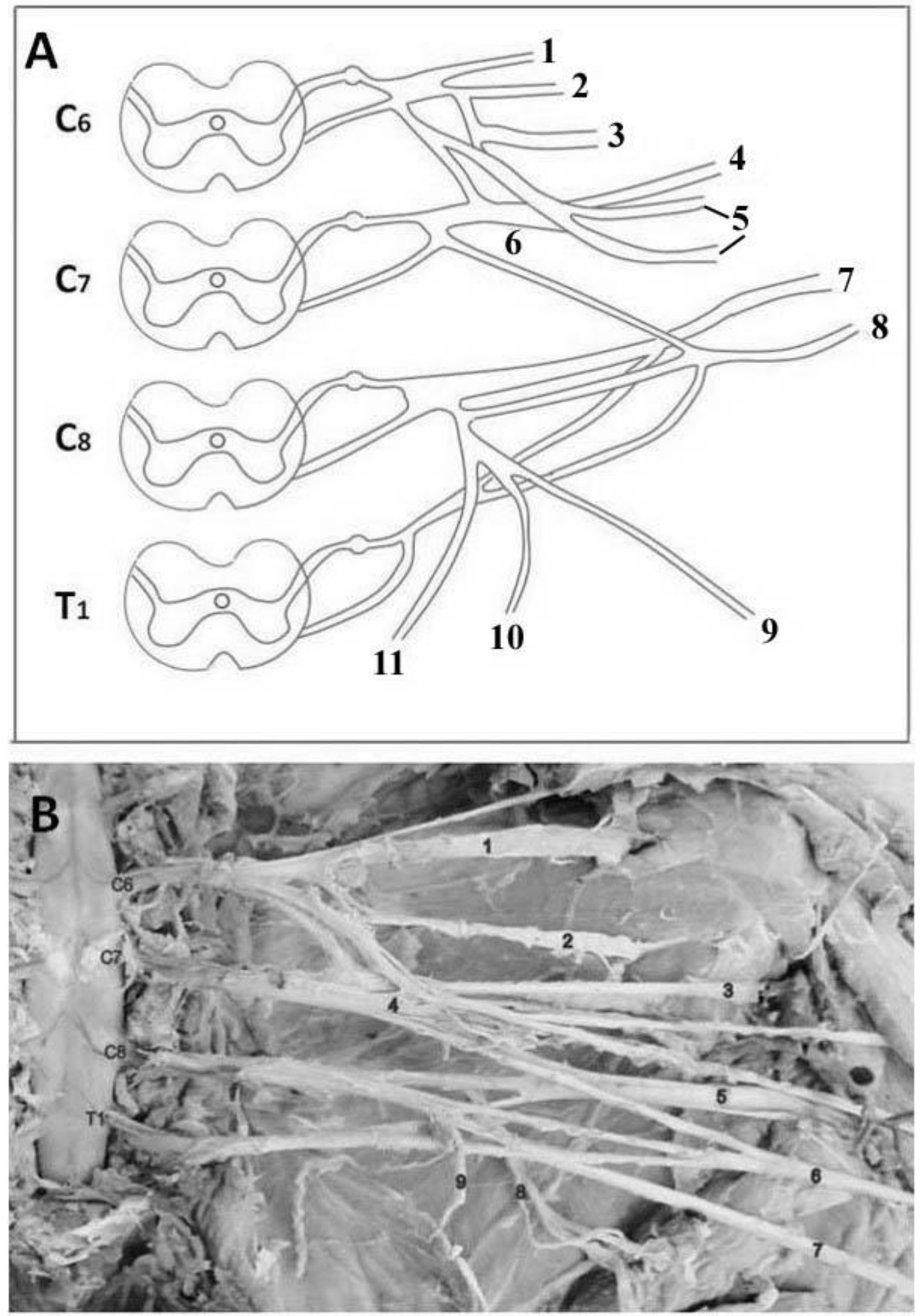


\section{Discussão}

Como descrito nos animais domésticos (GHOSHAL, 1986; DYCE et al., 2010), na chinchila (Chinchilla lanigera) (GAMBA et al., 2007), no lobomarinho (Arctocephalus australis) (SOUZA et al., 2010), no nutria (Myocastor coypus) (GUIMARÃES et al., 2013) e no veado catingueiro (Mazama gouazoubira) (VIEIRA et al., 2013), o plexo braquial da jaguatirica apresenta o segmento espinhal inicial a partir de C6. Diferem de roedores, como o rato (UZUN et al., 2001) e a paca (SCAVONE et al., 2008), os quais apresentam origem em C5 e, do observado nos marsupiais e nos lêmures (MILLER, 1934), nos monotremas (MILLER, 1934; KOIZUMI; SAKAI, 1996), nos gorilas, nos chimpanzés, nos gibões (KOIZUMI; SAKAI, 1995), nos macacos-de-cheiro (Saimiri sciureus) (ARAÚJO et al., 2012), nos macacos prego (Cebus apella) (RIBEIRO et al., 2005) e nas capivaras (FIORETTO et al., 2003), que apresentam o plexo braquial formado a partir de $\mathrm{C} 4$.

Com relação ao segmento espinhal final, nas jaguatirica e nas paca (SCAVONE et al., 2008), nos primatas do Novo e Velho Mundo (MILLER, 1934), nos monotremas (KOIZUMI; SAKAI, 1996), nos macacos-de-cheiro (ARAÚJO et al., 2012), nos macacos prego (RIBEIRO et al., 2005) e nos animais domésticos (GHOSHAL, 1986; DYCE et al., 2010; RICCI et al., 2013), este segmento corresponde a $T_{2}$, diferentemente do encontrado nos marsupiais e nos lêmures (MILLER, 1934), na capivara (FIORETTO et al., 2003), na chinchila (GAMBA et al., 2007), no lobo-marinho (SOUZA et al., 2010), na nutria (GUIMARÃES et al., 2013), e no veado catingueiro (VIEIRA et al., 2013), cujo segmento final corresponde a $\mathrm{T}_{1}$.

Os nervos componentes do plexo braquial da jaguatirica foram: subclávio, supraescapular, subescapular, musculocutâneo, peitorais craniais, axilar, radial, ulnar, mediano, toracodorsal e torácico lateral. No entanto, Freitas (2010) afirma que no gato doméstico, o plexo braquial é composto pelos nervos radial, mediano, ulnar, musculocutâneo, axilar e supraescapular, discordando com o que é preconizado por Done et al., (2002) o qual relata ainda a presença dos nervos subescapular e toracodorsal, à semelhança dos achados na jaguatirica, porém nenhum destes autores cita a presença do subclávio. Este nervo é citado como componente do plexo braquial apenas no macaco-decheiro (ARAÚJO et al., 2012).

De acordo com Zimpel et al. (2012), o graxaim do mato (Cerdocyon thous) e o graxaim do campo (Dusicyon gymnocercus) apresentam o plexo braquial relativamente semelhante ao da jaguatirica, apesar de terem início em $\mathrm{C}_{7}$ e não em $\mathrm{C}_{6}$ e terminarem em $\mathrm{T}_{1}$, o que também difere do cão doméstico, que possui o plexo braquial estendendo-se de $\mathrm{C}_{6}$ a $\mathrm{T}_{2}$ (DYCE; SACK; WENSING, 2010).

Além dos onze nervos descritos na jaguatirica, foi observado no lobo-marinho (SOUZA et al., 2010) o nervo peitoral caudal e torácico longo; no lobo-marinho, os peitorais (cranial e caudal) se originaram em direções opostas, sendo que o nervo peitoral cranial emergiu a partir do sexto nervo cervical e o peitoral caudal a partir do oitavo nervo cervical e do primeiro nervo torácico, assemelhando-se ao observado na jaguatirica para o peitoral cranial. O nervo torácico longo na chinchila (GAMBA et al., 2007) e no lobo-marinho (SOUZA et al., 2010) se formou a partir de ramificações dos sétimo e oitavo nervos cervicais, não sendo encontrado na jaguatirica. Na chinchila (GAMBA et al., 2007), o nervo peitoral cranial apresentou sua origem a partir de $\mathrm{C}_{7}$ e o nervo peitoral caudal a partir de $\mathrm{C}_{8} \mathrm{e}_{1}$, enquanto que o nervo torácico longo se mostrou proveniente de $\mathrm{C}_{7}$ e $\mathrm{C}_{8}$.

No macaco barrigudo (Lagothrix lagothricha) foi observado ainda o nervo torácico longo (CRUZ; ADAMI, 2010). Estes achados não correspondem aos encontrados na jaguatirica.

Quanto ao número de troncos nervosos do plexo braquial, foi possível constatar achados análogos ao da jaguatirica, em chinchila (GAMBA et al., 2007), em lobos-marinhos (SOUZA et al., 2010) e em macaco-decheiro (ARAÚJO et al., 2012), ou seja, todos apresentam quatro troncos, sendo que na chinchila e no lobosmarinhos, os dois primeiros troncos deixam o canal vertebral cranialmente às vértebras correspondentes. $\mathrm{O}$ terceiro nos lobos-marinhos tem origem entre a $\mathrm{C}_{7}$ e $\mathrm{T}_{1}$ e caudalmente a este ocorre a formação do quarto tronco que surge a partir do espaço entre a primeira e 
a segunda vértebra torácica, enquanto que na chinchila o terceiro e o quarto ocorrem caudalmente às vértebras correspondentes.

Diante do observado nas dissecações dos dois espécimes de jaguatirica, sugere-se que o plexo braquial destes animais é bastante semelhante ao dos carnívoros domésticos, bem como algumas espécies selvagens.

\section{Referências}

ARAÚJO, E. B.; LIMA, A. R.; PINHEIRO, L. L.; MUNIZ, J. A. P. C.; IMBELONI, A.; BRANCO, E. Origem e distribuição do plexo braquial de Saimiri sciureus. Pesquisa Veterinária Brasileira, Seropédica, v. 32, n. 12, p. 1351-1354, 2012.

BUDRAS, K. D.; McCARTHY, P. H.; FRICKE, W.; RICHTER, R.; HOROWITZ, A.; BERG, R. Anatomia do Cão - texto e atlas. 5 ed. São Paulo: Manole, 2012. 219 p.

CRUZ, G. A. M.; ADAMI, M. Anatomia do plexo braquial de macaco-barrigudo (Lagothrix lagothricha). Pesquisa Veterinária Brasileira, Seropédica, v. 30, p. 881-886, 2010.

DYCE, K. M.; SACK. W. O.; WESING, C. J. G. Tratado de anatomia veterinária. 4 ed. Rio de Janeiro: Guanabara Koogan, 2010. 840 p.

DONE, S. D.; GOODY, P. C.; EVANS, S. A.; STICKLAND, N. C. Atlas colorido de Anatomia veterinária do cão e do gato. Vol. 3. São Paulo: Manole, 2002. 10.16.

FIORETTO, E. T.; CASTRO, M. F. S.; GUIDI, W. L.; MAINARDI, R.; DE SOUZA, R. R.; RIBEIRO, A. A. C. M. Gross anatomic organization of the capybara's brachial plexus (Hydrochaeris hydrochaeris). Anatomia, Histologia, Embryologia, Berlin, v. 32, p. 169-174, 2003.

FREITAS, A. I. A. Regeneração espontânea da lesão do plexo braquial no gato: Relato de caso. PUBVET, Londrina, v. 4, n. 42, Ed. 147, Art. 990, 2010.

GAMBA, C. O.; CASTRO, T. F.; RICKES, E. M.; PEREIRA, M. A. M. Sistematização dos territórios nervosos do plexo braquial em chinchila (Chinchilla lanigera). Brazilian Journal of Veterinary Research and Animal Science, São Paulo, v. 44, n. 4, p. 283-289, 2007.

GHOSHAL, N. G. Nervos espinhais. In: SISSON, S; GROSSMAN, J. D. (Ed.) Anatomia dos animais domésticos. 5 ed. Rio de Janeiro: Guanabara Koogan, 1986. p. 1597-1600.

GUIMARÃES, R. T.; BIRCK, A. J.; FILADELPHO, A. L.; GUIMARÃES, G. C. B ARCELOS, R. P. Origem e distribuição do plexo braquial no nutria (Myocastor coypus). Revista Científica Eletrônica de Medicina Veterinária, Garça, n. 20, 2013.

INTERNATIONAL COMMITTEE ON VETERINARY GROSS ANATOMICAL NOMENCLATURE. Nomina anatômica veterinária. 5 ed. Knoxville: World Association on Veterinary Anatomist, 2012. 160 p.
KOIZUMI, M.; SAKAI, T. The nerve supply to coracobrachialis in apes. Journal of Anatomy, London, v. 186, p. 395-403, 1995.

KOIZUMI, M.; SAKAI, T. On the morphology of the brachial plexus of the platypus (Ornithorhynchus anatinus) and the echidna (Tachyglossus aculeatus). Journal of Anatomy, London, v. 190, p. 447-455, 1996.

KÖNIG, H. E.; LIEBICH, H. J. Anatomia dos animais domésticos. Porto Alegre: Artmed, 2011. 787 p.

MILLER, M. E.; CHRISTENSEN, G. C.; EVANS, H. E. Anatomy of the dog. Philadelphia: W. B. Saunders, 1964. 746 p.

MILLER, R. A. Comparative studies upon the morphology and distribution of the brachial plexus. American Journal of Anatomy, New York, v. 54, p. 143-175, 1934.

MOURA, C. E. B.; ALBUQUERQUE, J. F. G.; MAGALHÃES, M. S.; SILVA, N. B.; OLIVEIRA, M. F.; PAPA, P. C. Análise comparativa da origem do plexo braquial de catetos (Tayassu tajacu). Pesquisa Veterinária Brasileira, Seropédica, v. 27, p. 357-362, 2007.

MURRAY, J. L.; GARDNER, G. L. Leopardus pardalis. Mammalian Species - American Society of Mammalogists, Northampton, n. 548, p. 1-10, 1997.

OLIVEIRA, T. G.; CASSARO, K. Guia de identificação dos felinos brasileiros. São Paulo: Sociedade de Zoológicos do Brasil, 1999. $60 \mathrm{p}$.

RIBEIRO, A. R.; PRADA, I. L. S.; SILVA, Z.; BARROS, R. A. C.; SILVA, D. C. O. Origem do plexo braquial do macaco Cebus apella. Brazilian Journ al of Veterinary Research and Animal Science, São Paulo, v. 42, n. 2, p. 143-149, 2005.

RICCI, D. G.; GUAZZELLI FILHO, J.; PINTO E SILVA, J. R. C.; MATHEUS, S. M. M.; FILADELPHO, A. L. Plexo braquial de mamíferos e aves - revisão de literatura. Revista Científica Eletrônica de Medicina Veterinária, Graça, n. 20, 2013.

SCAVONE, A. R. F.; MACHADO, M. R. F.; GUIMARÃES, G. C.; OLIVEIRA, F. S.; GERBASI, S. H. B. Análise da Origem e Distribuição dos nervos periféricos do plexo braquial da paca (Agouti paca, linnaeus, 1766). Ciência Animal Brasileira, Goiânia, v. 9, n. 4, p. 1046-1055, 2008.

SOUZA, D. A. S.; CASTRO, T. F.; FRANCESCHI, R. C.; SILVA FILHO, R. P.; PEREIRA, M. A. M. Formação do plexo braquial e sistematização dos territórios nervosos em membros torácicos de lobos-marinhos Arctocephalus australis. Brazilian Journal of Veterinary Research and Animal Science, São Paulo, v. 47, n. 2, p. $168-174,2010$.

UZUN, A.; CENGIZ, N.; KAVAKLI, A.; KARAKAS, S. Morphological and microscopical examination of the rat brachial plexus. Turk-Veterinerlik-ve-Hayvancilik-Dergisi, Ankara, v. 25, p. 397-402, 2001.

VIEIRA, L. G.; RIBEIRO, P. R. Q.; LIMA, M. O.; SOUZA, R. R.; VALDES, S. A. C.; SANTOS, A. L. Q. Origens e ramificações do plexo braquial do veado catingueiro Mazama gouazoubira (Artiodactyla: Cervidae). Biotemas, Florianópolis, v. 26, n. 1, p. 137-146, 2013. 